\title{
Outcome of mid- and lower face lifting using bidirectional cone sutures at 6 months and 1 year
}

Phoebe Kar Wai Lam, MBChB (Otago), MRCS (Edin), MSc PD (Cardiff)iD, James Yui Lam, MBBS (HK), MRCSED, PGDIPCLINDERM(D, Alvin Kar Wai Lee, MBChB (CUHK), DCH (Sydney), Dip Derm (Glasgow), Ms Clin Derm (Cardiff), Ms PD (Cardiff) (iD, Clyde Wang Lung Luk, MBBS, MSc, FCOHK, FCSHK(D, Paul Man Kei Tam, MBBS (HKU), MRCP (UK), FHKAM, FHKCP, DDME (CUHK), DPD (D), Cheuk Hung Lee, MBBS (HK), FHKAM (MED), FHKCP, MSc PD (Cardiff), MRCP (UK), DPD (Wales), Dip Derm (Glasgow), PG Dip Clin Derm (London), MRCP (London), Grad Dip Derm (NUS), Dip Med (CUHK) iD

Association of Doctors in Aesthetic Medicine, Hong Kong

\begin{abstract}
Soft tissue ptosis occurs with age, leading to loss of facial volume. The facial retaining ligamentous system is an intricate network of fibrous tissues connecting the whole face, restraining soft tissues from gravitational forces, and opposing facial movements. With proper positioning and anchorage within the ligamentous grid and superficial fat repositioning, lifting the mid-face and adjacent areas (e.g., jowl) is likely to occur. We aimed to determine whether inserting 3 pairs of bidirectional cone sutures can effectively improve the nasolabial fold (mid-face) and jowl (lower face) in Asian patients with mild to moderate mid-face laxity at 6 months and 12 months. Ten healthy volunteers with mild to moderate facial laxity received 3 pairs of bidirectional cone threads, inserted in a straight parallel technique. Based on validated evaluation parameters: facial laxity rating scale (FLRS) and the wrinkle severity rating scale, a total of 7 independent reviewers, among which 3 assessed the change in mid-face laxity (nasolabial fold), and the other 4 reviewers evaluated the change in jowl or lower face contour based on FLRS at 6 months and 12 months. All reviewers were experienced esthetic doctors. According to the reviewers' evaluation based on the validated parameters, there was a linear improvement in the mid-face laxity/nasolabial fold and lower face laxity/jawline contour self-reporting patients' satisfaction at 6 months and 12 months compared to baseline in all 10 patients. There was continuous improvement in mid-face laxity and lower face laxity from baseline, 6 months, and 12 months after treatment. The evaluation results among the reviewers were similar, apart from reviewer 7 . All patients gave their best rating at 12 months, and their satisfaction almost doubled compared to that before treatment.

Level of Evidence: Level IV, brief communication
\end{abstract}

Keywords: aging; fascia; ligaments; nasolabial fold; rhytidoplasty

\section{Introduction}

Facial soft tissues are held in their natural position against gravity by the retaining ligament system [1]. With age and loss of deep fat volume, superficial fat compartments slide under the force of muscle retraction and sag with gravity, although curbed by the retaining ligaments (false), resulting in deepening of the nasolabial fold. The descent of the superficial fat compartments (nasolabial, superior, and inferior jowl fat pads) is most commonly responsible for patients' concerns, especially the nasola-

Received May 24, 2021; Revised June 8, 2021; Accepted June 8, 2021

Corresponding author: Phoebe Kar Wai Lam

E-mail: myperfectskinsolution@gmail.com

This is an Open Access article distributed under the terms of the Creative Commons Attribution Non-Commercial License (http://creativecommons.org/licenses/by-nc/4.0), which permits unrestricted non-commercial use, distribution, and reproduction in any medium, provided the original work is properly cited.

Copyright $@ 2021$ Korean Society of Korean Cosmetic Surgery and Medicine (KSKCS \& KCCS). 


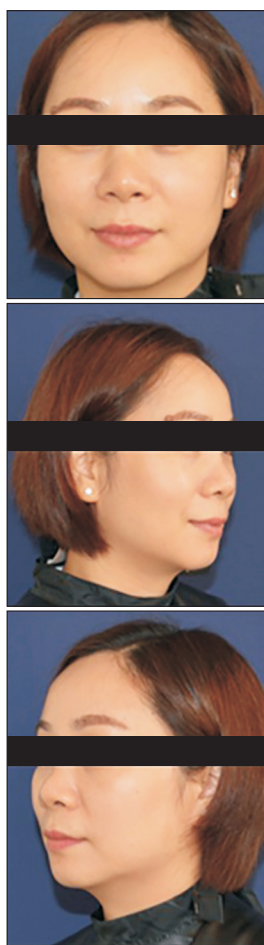

Baseline

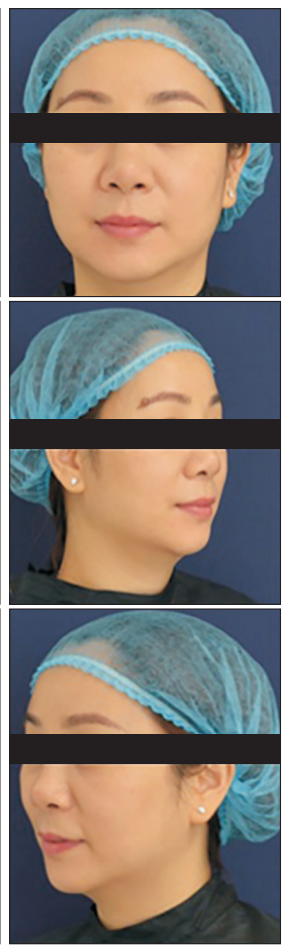

6 months

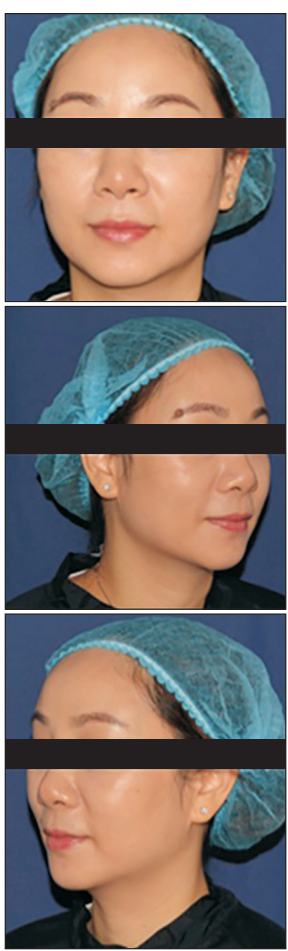

12 months

Fig. 1. Clinical photo (central, top; right lateral, middle; left lateral, bottom) of patient taken at baseline line, 6 months, and 12 months.

bial folds (NLF), marionette lines, and jowls.

Bidirectional cone sutures are composed of a monofilament made of $82 \%$ poly L-lactic acid (PLLA) and $18 \%$ polyglycolic acid (PLGA) (Fig. 1). These sutures are inserted to reposition the descended superficial fat pad (mobile) via anchorage within the retaining ligaments (fixed), improving facial laxity in patients with mild to moderate facial laxity.

In an earlier paper, we established a significant improvement in mid-face laxity by 3 months following this insertion technique. In this study, we determined at 6 months and 12 months follow-up, if there was evidence of any sustained midface lifting and any lower face (jowl), laxity improvement was observed.

\section{Case report}

Ten healthy volunteers with mild to moderate facial laxity with no significant facial volume loss received 3 pairs of bidirectional cone threads inserted in a straight parallel technique in the mid-face. There were 9 females and 1 male, aged 24 years to 53 years (average, 42.4 years).

There were 7 independent reviewers ( 3 for mid-face and 4 for lower face) all of whom were local experienced esthetic doc- tors. The mid-face reviewers assessed the mid-face laxity based on the validated facial laxity assessment parameter using the wrinkle severity rating scale (WSRS) for NLF and facial laxity rating scale (FLRS), while the lower-face reviewers evaluated the lower face laxity based on FLRS only.

All 10 volunteers were required to complete a subjective selfsatisfaction questionnaire using the Global Esthetic Improvement Scale (GAIS) and the individual subjective satisfaction rating scale (SSRS) during each follow-up visit.

Procedure: Three pairs of $30 \mathrm{~cm}$ absorbable sutures ( 8 cones) were inserted in straight parallel vectors in the mid-face of each participant.

Pre-marking before procedure: The last cone of the distal end anchored within the superficial fat pads produced the most efficient pulling point (EPP) for maximal face lifting. The entry and exit points (approximately $6 \mathrm{~cm}$ and $12 \mathrm{~cm}$ proximal to the EPP, respectively) were marked in the sitting position.

Technique: The face was sterilized, followed by local infiltration of Xylestesin-A (1:80,000; 3M ESPS, Neuss, Germany) at each pre-marked entry and exit point.

An 18G needle was used to create a small skin puncture at each of the 3 pre-marked entry points on each side of the face. The first of the 2 sharp needles $(23 \mathrm{G} 12 \mathrm{~cm}$ ) of each 8-cone suture was inserted vertically into the subcutaneous tissue via their respective skin puncture, as far as the 5-mm marking on the needle, then pivoted 90 as the needle tunneled subdermally towards the EPP (distally) and exited through the skin. The second of the 2 sharp needles of each 8-cone suture entered vertically through the same entry point, as far as the $5 \mathrm{~mm}$ needle marking, then tunneled along the subdermal plane in the opposite direction, toward the proximal pre-marked exit point. This (upper) part of the 8-cone suture was anchored within the relatively fixed (area close to the zygomatic ligament) to support the sagging soft tissue that advanced and engaged over the lower half of the suture [2]. These cones were gently manipulated so that each cone locks over its corresponding knot on the same suture. Excess suture lengths were trimmed and removed when the desired effects were achieved.

\section{Results}

For all patients, there was an overall improvement of facial contours. In addition, one of the patients' clinical photographs before treatment, 6 months and 12 months after treatment is shown in Fig. 2. 


\section{Mid-face}

The mean score analysis results from 3 independent reviewers showed a linear improvement from baseline to 6 months and 12 months after treatment using the FLRS and WSRS (Fig. 2). Improvement based on the WSRS was significant at 6 months. The 3 reviewers' responses were consistent in the rating of these patients, with a standard deviation of 0.5 .

\section{Lower face}

There was also a linear improvement in the lower face laxity from baseline to 6 months and 12 months (Fig. 3). However, the standard deviation in response among the reviewers was approximately 1 , as one of the reviewers' ratings was very different compared to the other 3 reviewers.

\section{Patients' satisfaction}

Regarding patient satisfaction, all patients showed improvement at 6 months and 12 months. They gave the best rating at 12 months in the GAIS (Fig. 4), and satisfaction was doubled compared to baseline, as determined by the Self-Satisfaction Rating Scale (SSRS) (Fig. 5). Common side effects, such as bruising, swelling, and tenderness at the treatment site, have

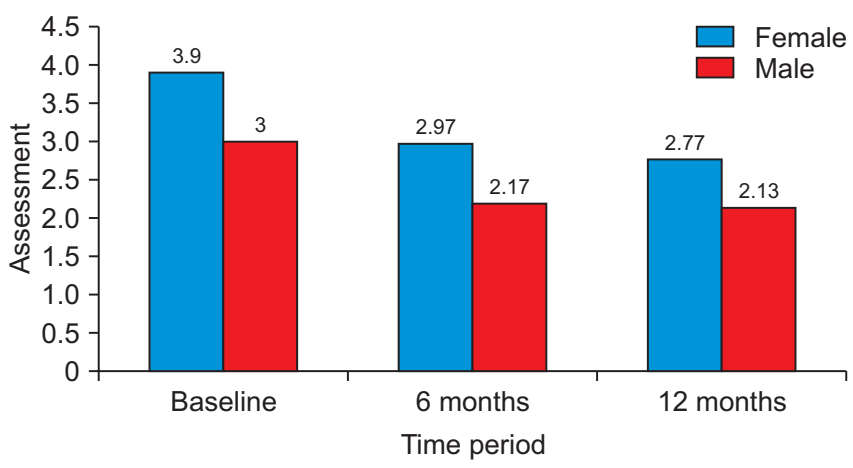

Fig. 2. Average mid-face assessment by reviewers 1-3.

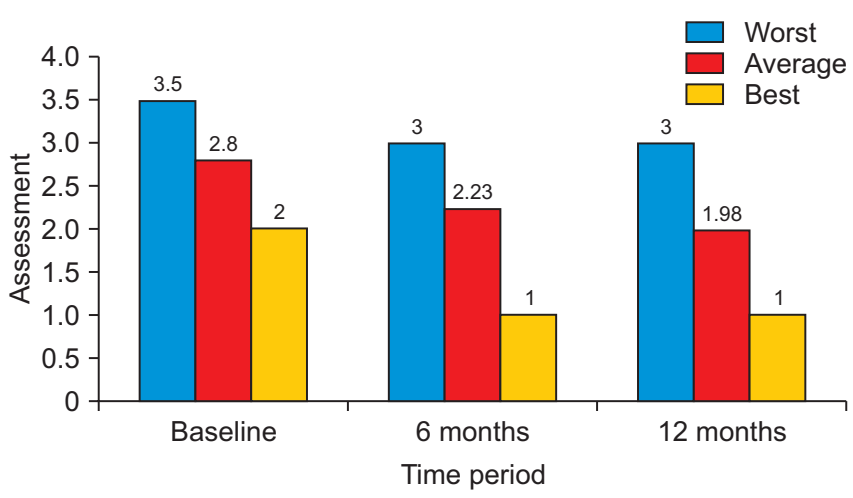

Fig. 3. Lower face assessment by reviewers 4-7. been reported, although mostly resolved by 24 to 48 hours.

\section{Discussion}

A previous study established that following placement of sutures within the subdermal layer, the implanted PLLA/PLGA will stimulate new collagen synthesis (predominantly type-1 collagen), which starts approximately 3 months, and the builtup of the new collagen will eventually form a collagen capsule around the cone, which reinforces the suture fixation to the surrounding soft tissues. This process was continued for 24 months [3]. This was consistent with our observation in the participants who demonstrated distinct improvement in mid-face laxity and NLF within 3 months following placement of the cone sutures in our patients. This mid-face improvement was significant at 6 months and was sustained at 12 months follow-up.

Although the cone's sutures are arranged within the midface, 3 of our 4 reviewers concurred that there was an improvement in the lower face laxity. Further analysis revealed a linear improvement in the lower facial contour following suture placement at 6 months and 12 months. The observed effect is likely related to a combination of factors.

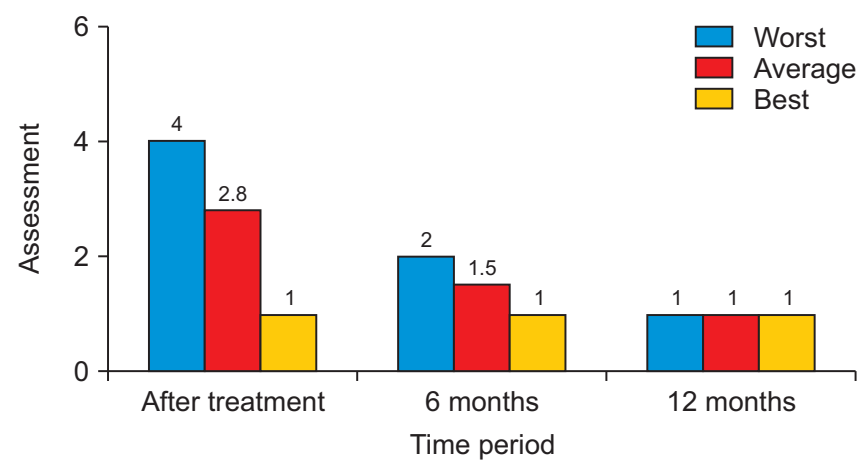

Fig. 4. Global Esthetic Improvement Scale (GAIS) ratings.

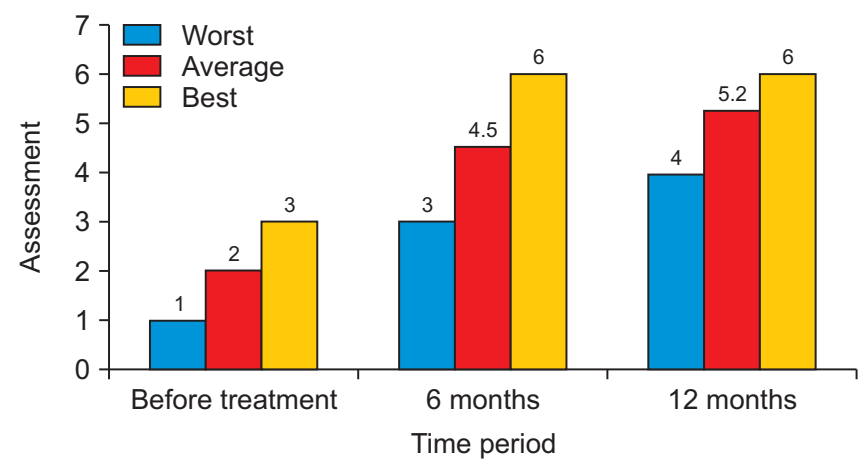

Fig. 5. Self-satisfaction rating scale (SSRS). 
First, all our healthy volunteers were young and had only mild to moderate mid-face laxity according to the FLRS with adequate skin elasticity; thus, capable of adequate collagen synthesis following thread placement.

Second, the freely moving hollow cone design on these bidirectional sutures provided a larger surface area for strong tissue engagement and efficient repositioning of the descended superficial fat system immediately after placement.

Third, soft tissue engagement was reinforced by the freefloating cones that lock behind the intercalated knots and the gathering of the descended tissue (superficial fat, nasolabial, and jowl) following placement to the point where soft tissue engagement "clicks" over the cones.

Finally, the exit points were selected to ensure strong anchorage (the top end was placed in the fibrous and less mobile, close to the zygomatic ligament, true retaining ligament) with the most EPP distally (within the targeted ptotic superficial fat) that serves to counteract daily repetitive facial movements and gravity.

Additionally, suture placement will further enhance the improved lower facial jawline, especially when placed according to the straight-line vector placement, that is, perpendicular to the area of the lower face where it needs to be lifted. No major discomfort or side effects (e.g., transient bruising, swelling, and pain) have been reported during this time.

Midface placement of the bidirectional cone suture thread can provide steady lifting in the mid-face and some lower face improvement at 6-month and 12-month follow-up. Patient sat- isfaction was doubled (SSRS) by a year.

\section{Acknowledgments}

I would like to express profound gratitude to Dr. Edwin Lau for his valuable and constructive suggestions. His expertise and availability are been greatly appreciated.

I would also like to thank the staff, especially Claire, David, and Christina of Clovers Medical Technology Ltd. for their assistance during this study period.

\section{Conflicts of interest}

All bidirectional cone and knot sutures were supplied by Clover Medical Technology (HK) Limited. No other financial relationship with any pharmaceutical companies to disclose.

\section{References}

1. Furnas DW. The retaining ligaments of the cheek. Plast Reconstr Surg 1989;83:11-6.

2. Lorenc ZP, Goldberg D, Nestor M. Straight-line vector planning for optimal results with silhouette instalift in minimally invasive tissue repositioning for facial rejuvenation. J Drugs Dermatol 2018;17:786-93.

3. Russo PR, Vercesi F, Migliorini L, Christen MO. Histological findings after insertion of PLLA sutures with bi-directional cones in humans: two years follow-up. J Plast Path Dermatol 2018;14:121-5. 